model, there was statistically significant decline in total scores of seizures in both the experimental test drug groups when compared with the control group for MES model $(p<0.01)$. The above said parameter was in comparison to the standard drug in group VI with respect to the experimental test drug groups (groups VII and VIII, respectively, with [p > 0.05]).

Conclusion: The benzimidazole group of drugs had significant anticonvulsant properties in experimentally induced animal models.

\section{A0020: Evaluation of Anticonvulsant Activity of Propranolol by using Electrically Induced Animal Models Chavan M.D., ${ }^{1}$ Karamthoti M.B., ${ }^{2}$ Kurra S.B. ${ }^{1}$ \\ ${ }^{1}$ Department of Pharmacology, MIMS, Tamil Nadu, India \\ ${ }^{2}$ Department of Physiology, MIMS, Tamil Nadu, India}

Objective: To evaluate the anticonvulsant activity of propranolol in Wistar albino rats by maximal electroshock (MES)-induced seizure model.

Methods: The study was approved by the Institutional Animal Ethics Committee (IAEC). The study was conducted in accordance with the CPCSEA guidelines. Healthy, adult Wistar albino rats of either sex weighing between 180 and $250 \mathrm{~g}$ were used for the study. The animals were procured from the central animal house and were acclimatized in the experimental laboratory for 7 days. The study consisted of three groups with six animals in each group. Group I: control (equivalent volume of normal saline, i.p.); group II: diphenylhydantoin (25 mg/kg BW, i.p.); group III: propranolol (i.p.). Anticonvulsant activity in Wistar albino rats was assessed by MES model. The data were expressed as median \pm SE. Statistical significance among study groups was carried using Graph Pad Instat Software, by ANOVA test followed by Bonferroni's post hoc test.

Results: In group II (standard) all animals were protected by absence of THLE when compared with group I (control). Group II also exhibited significant decline in scores when compared with control group. Administration of propranolol in groups III also showed significant percent decline in THLE as well as scores when compared with group I. Percent decline in THLE and scores in group III were comparable to the group II.

Conclusion: Propranolol exhibits significant anticonvulsant activity in MES model in Wistar albino rats.

\section{A0021: Screening of Antiseizure Activity of Aryl Acetic Acid Compounds in Wistar Albino Rats by using PTZ and MES Tests \\ Chavan M.D., ${ }^{1}$ Karamthoti M.B., ${ }^{2}$ Kurra S.B. ${ }^{1}$ \\ ${ }^{1}$ Department of Pharmacology, MIMS, Tamil Nadu, India \\ ${ }^{2}$ Department of Physiology, MIMS, Tamil Nadu, India}

Objective: To screen the antiseizure activity of aryl acetic acid compound in Wistar albino rats using PTZ and MES tests.

Methods: After obtaining the approval from Institutional Review Board (IRB), the healthy adult Wistar albino rats of either sex weighing between 180 and 250 g were procured from the central animal house of the Institution. The CPCSEA guidelines were followed for conducting the experiments on animals. The animals were housed in the research laboratory for 1 week before initiation of study. The animals were then randomly selected and divided into control, standard, and test groups $(n=6)$. Group I: control for PTZ; group II: standard for PTZ (sodium valproate, i.p.); group III: aryl acetic acid compound for PTZ (i.p.); group IV: control for MES; group V: standard for MES (diphenylhydantoin, i.p.); and group VI: aryl acetic acid compound for MES (i.p.). One-way ANOVA with Bonferroni's post hoc test was used for statistical significance.

Results: The standard drug sodium valproate in group II and test drug aryl acetic acid compound in group III showed significant reduction in onset, duration, and number of seizures when compared with the group I (control). Similarly, the standard drug diphenylhydantoin in group $\mathrm{V}$ and test drug aryl acetic acid compound in group VI showed significant reduction in scores of seizures and THLE when compared with the group IV (control).

Conclusion: The experimental drug aryl acetic acid compound had significant antiseizure activity in both PTZ and MES tests in Wistar albino rats.

\section{A0022: Peroxisome Proliferator Activated Receptor $\sigma$ $(\mathrm{pPAR} \sigma)$ Agonists and Their Role on Epilepsy-Induced Seizures: An Experimental Evaluative Study} Chavan M.D., ${ }^{1}$ Karamthoti M.B., ${ }^{2}$ Kurra S.B. ${ }^{1}$ ${ }^{1}$ Department of Pharmacology, MIMS, Tamil Nadu, India ${ }^{2}$ Department of Physiology, MIMS, Tamil Nadu, India

Objective: To evaluate the antiepileptic properties of peroxisome proliferator activated receptor $\sigma(p P A R \sigma)$ agonists in chemically and electrically induced seizures in experimental laboratory animal models.

Methods: Prior to the experimentation on animals, the research proposal was approved by the appropriate official bodies (Institutional Research Committee [IRC] and Institutional Animal Ethics Committee [IAEC]). The research protocol followed the guidelines as per the directions from the INSA and CPCSEA throughout the study period. The study had a total of eight groups and two animal models each within four groups. Groups I to IV were for chemically induced seizure model and groups V to VIII for electrically induced model. There were vehicle control, standard, and two experimental test drugs belonging to PPAR $\sigma$ agonists for groups I to IV and groups V to VIII for chemically and electrically induced seizure models, respectively. The data were analyzed using appropriate statistical test methods and the p-value of less than 0.05 was considered to be statistically significant.

Results: In this study, there were a total of six parameters (four for chemically induced seizure model and two for electrically induced seizure model) which accounted for determining the statistical difference among control, standard, and experimental test drug groups. In this study, it was found that there was a difference between the experimental test drug groups PPAR $\sigma$ agonists+ in comparison to the control group for number, score, onset, and duration of seizures in chemically induced seizure model ( $p$-value more than 0.05). Statistical analysis also confirmed that there was a statistically significant difference between the standard (group II) and 
experimental test drug groups (i.e., groups III and IV) with p-value of less than 0.05 . In another model with electrically induced seizure test, Results were of similar nature as the chemically induced seizure model, as in both the experimental test drug groups PPAR $\sigma$ agonists+, that is, groups VII and VIII, there was a slight reduction in score of seizures, when compared with the group V (standard) with p-value of more than 0.05 .

Conclusion: Peroxisome Proliferator Activated Receptor $\sigma(p \mathrm{PAR} \sigma)$ agonists showed increase in onset with decline in duration, number, and score of seizures in chemically, as well as decline in score of seizures in electrically induced seizures in experimental laboratory animal models.

\section{A0023: Impact of Repeated Health Education Delivery on Antiepileptic Drug Adherence in People with Chronic Epilepsy in the Community \\ Karan Chouhan, ${ }^{1}$ Chirag Gupta, ${ }^{1}$ Archita Chawla, ${ }^{1}$ \\ Sandeep Kaushal, ${ }^{1}$ Gagandeep Singh, ${ }^{1}$ Namita Bansal, ${ }^{1}$ \\ Suman Sharma ${ }^{1}$ \\ ${ }^{1}$ Dayanand Medical College and Hospital, Ludhiana, Punjab, India}

Introduction: Epilepsy is a chronic disorder with long-term treatment, and adherence to antiepileptic drugs decreases with time. Repeated counseling with adherence reinforcement may prevent this adherence decay.

Objective: To study the impact of repeated health visits and patient education on adherence to antiepileptic drugs in people with chronic epilepsy in the community.

Methods: The setting of the study was provided by community care trial of home-based care delivered by primary health care workers versus routine clinic-based care. We studied the association between monthly pill counts and self-reported adherence questionnaire score with age, gender, religion, ethnic origin, education, occupation, monthly family income, and socioeconomic class. In addition, we created a dummy variable, which represented baseline scores for each subject.

Results: There was considerable inter individual variations on pill count and SRMS score. Overall, lag SRMS, religion, ethnic origin, and monthly family income determined adherence status. A steady increase in the proportion of people with appropriate pill count was observed in the home-based care group but not in the clinic-based care group.

Conclusion: Treatment adherence varies considerably between individuals and is influenced by individual behavior. However, repeated adherence reinforcement by primary health care workers may lead to improvement in adherence.

\section{A0024: EEG Changes in Autoimmune Neuronal Synaptic Encephalitis-A Case Study \\ Laxmi Khanna ${ }^{1}$, Nandini Agarwal ${ }^{1}$ \\ ${ }^{1}$ Department of Neurophysiology, Sir Ganga Ram Hospital, New Delhi, India}

Introduction: Autoimmune neuronal synaptic encephalitis is a group of antibody-mediated inflammatory diseases of the central nervous system. We have used bedside EEG recording in the early diagnosis of suspected cases of autoimmune encephalitis.
Methods: The EEG records of 15 patients $(\mathrm{M}: \mathrm{F}=10: 5)$ with suspected autoimmune encephalitis were studied. Their clinical profile, antibody studies, MRI findings, and outcome were also recorded.

Results: $-26.67 \%$ patients had periodic discharges in the EEG, 6.67\% patients had triphasic complexes, $20 \%$ patients had a generalized delta slowing with delta brushes, $20 \%$ patients had generalized epileptiform discharges, $13.72 \%$ had a nonconvulsive status epilepticus, and $13.72 \%$ had a rhythmic delta slowing in the EEG.

Conclusion: EEG is an inexpensive tool in the early diagnosis of suspected autoimmune neuronal synaptic encephalitis. The Results of an abnormal EEG will aide in the diagnosis and -rapid initiation of treatment even before other test Results become available. Often, antibody studies do not correlate with the clinical presentation and bedside EEG could be an invaluable guide in clinically suspected cases. Initiation of treatment early in the illness improves the clinical outcome and delays the long-term sequelae.

\section{A0025: Subjective Memory Complaints in Temporal Lobe Epilepsy What Contributes to an Experience of Memory Dysfunction? \\ Desai M., ${ }^{1}$ Rajeshree S., ${ }^{1}$ Sapre A., ${ }^{1}$ Kalika M.,, ${ }^{1}$ Shah U., ${ }^{1}$ Ravat $\mathrm{S} .^{1}$ \\ ${ }^{1}$ Department of Neurology, King Edward Memorial Hospital, Mumbai, Maharashtra, India}

Patients with temporal lobe epilepsy (TLE) often present with subjective memory complaints (SMC) that cause significant distress and impact their quality of daily life (Giovagnoli and Avanzini, 2000). Sometimes, these complaints do not correlate with Objective memory test (OMT) scores (Hall et al, 2009). Memory deficits are important variables in planning treatment and predicting surgical outcome. Hence, identifying factors that influence SMC helps in planning effective treatment.

Objective: To delineate predictors of subjective memory complaints.

Methods: Retrospective data analysis of 97 adult patients who had (1) left mesial TLE, (2) underwent comprehensive neuropsychological examination, (3) IQ > 70. A stepwise binary logistic regression was conducted using data from clinical history and test scores for independent variablesdemographics (age, gender, education, and occupation), intelligence, memory tests (R-AVLT, paired associates and complex figure test), seizure, medication, mood, and psychosocial factors (stigma and overprotection) as predictors of SMC.

Results: Seventy-five patients (77.31\%) reported SMC, of which one fourth (25.33\%) showed no impairment on any OMT. The regression model indicated two significant predictors of SMC, mood issues and one memory test (paired associates) score, $\chi^{2}=14.93, \mathrm{p}<0.001$.

Conclusion: The odds of reporting SMC increased with the presence of mood issues and poor performance on one memory test. Mood issues appear to be an important factor impacting memory significantly and resulting in subjective experience of problems despite no impairment in most Objective tests. It is important to elicit subjective 